\title{
Characterization of Liver Disease using Image Texture Analysis
}

\author{
Babkir A. Awad Alla ${ }^{1}$, Abdoelrahman Hassan A. B ${ }^{1}$, Mohamed Elfadil M. Gar-elnabi ${ }^{1}$, Asma I. Ahmed ${ }^{1}$ \\ ${ }^{1}$ Collage of Medical Radiologic Science, Sudan University of Science and Technology, Khartoum, Sudan
}

\begin{abstract}
This study aimed to use the texture analysis and classification methods to characterize the normal liver and liver pathologies in US images using image processing program (IDL, interactive data language). A prospective study for 50 patient with normal liver and same number of patient with hepatitis $B, C$ and liver cirrhosis was examined using ultrasound scan after successful laboratory investigation and diagnostic confirmation also. Ultrasound images was acquired using mobile GE LOGIQ-5, US scanner and the image extracted and treated for image processing analysis using interactive data language. Tiff format was created as IDL variables and then using 3x3 window the image was scanned and based on the image histogram the selected feature also called FOS was calculated using this window. Linear discriminant analysis was used for the tissue classification. The study found that the liver cirrhosis, hepatitis (B), and $(C)$ texture reveal a different underlying pattern compared to the normal liver tissues with classification sensitivity and specificity $97.1 \%, 69.8 \%, 73.3 \%$, and $92.5 \%$ respectively, and the combination of the texture features throughout the different ultrasound images provide the highest predictive overall accuracy of $84.9 \%$ using linear discriminant analysis.
\end{abstract}

Keywords: Liver, hepatitis, cirrhosis, texture analysis, first order statistics.

\section{Introduction}

The liver lies in the right upper quadrant of the abdomen, suspended from the right hemi diaphragm. Functionally, it can be divided into three lobes: right, left, and caudate. The right lobe of the liver is separated from the left by the main lobar fissure, which passes through the gallbladder fossa to the inferior vena cava (IVC). The right lobe of the liver can be further divided into anterior and posterior segments by the right intersegmental fissure. The left intersegmental fissure divides the left lobe into medial and lateral segments.

Also liver can be divided into many segments; which are Segment I is the caudate lobe, segments II and III are the left superior and inferior lateral segments, respectively, and segment IV, which is further divided into IVa and IVb, is the medial segment of the left lobe. The right lobe consists of segments V and VI, located caudal to the transverse plane, and segments VII and VIII, which are cephalad. (Sugar-baker PH, 1988 and Soyer P et.al 1994).
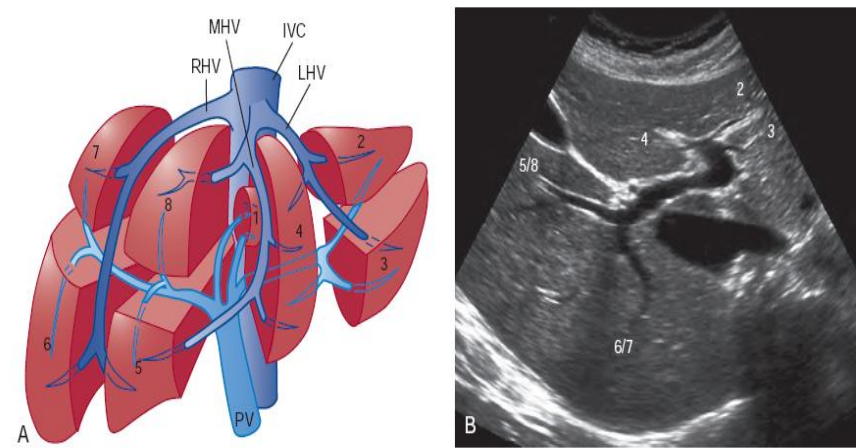

Figure 1: Couinaud's functional segmental anatomy (From Sugarbaker PH, 1988)

Ultrasound is one of the basic diagnostic tools that used to diagnose a variety of liver disease using its good textural properties as well as it can be used for accurate measurement of liver dimensions Gosink and Leymaster (1981) proposed measuring the liver length in the mid-hepatic line. In $75 \%$ of patients with a liver length of greater than $15.5 \mathrm{~cm}$, hepatomegaly is present. Niederau C, et.al 1983) measured the liver in a longitudinal and anteroposterior diameter in both the mid clavicular line and the midline and correlated these findings with gender, age, height, weight, and body surface area.

Viral hepatitis is a common disease that occurs worldwide. It is responsible for millions of deaths secondary to acute hepatic necrosis or chronic hepatitis, which in turn may lead to portal hypertension, cirrhosis, and hepatocellular carcinoma (HCC). Hepatitis was subcategorized in three common type and other categories also which are; Hepatitis $\mathrm{A}$ is an acute infection leading to complete recovery or death from acute liver failure. Hepatitis $\mathrm{B}$ is transmitted parenterally, as well as by non-percutaneous exposure through sexual contact and at birth. The two most useful markers for acute infection are hepatitis B surface antigen (HBsAg) and antibody to hepatitis B core antigen (anti-HBc). (Stephanie R. et.al 2011). Hepatitis C (predominantly) and hepatitis $\mathrm{E}$ were formerly called non-A, non-B (NANB) hepatitis, first recognized in 1974. U.S. investigators were surprised to learn that the majority of cases of posttransfusion hepatitis were not secondary to hepatitis B but to an unknown virus or viruses.

Hepatitis D, or delta hepatitis, is entirely dependent on HBV for its infectivity, requiring the $\mathrm{HBsAg}$ to provide an envelope coat for the hepatitis D virus (HDV). Its geographic distribution is therefore similar to that of hepatitis B. 29 (Davis GL. 1996).

The sonographic features parallel the histologic findings. The liver parenchyma may have a diffusely decreased echogenicity, with accentuated brightness of the portal triads or periportal cuffing. Hepatomegaly and thickening of the gallbladder wall are associated findings (Wilson SR. 2004) 


\section{International Journal of Science and Research (IJSR) \\ ISSN (Online): 2319-7064}

Index Copernicus Value (2013): 6.14 | Impact Factor (2014): 5.611

In most patients the liver appears normal. (Zwiebel WJ. et.al 1995). Most cases of chronic hepatitis are also sonographically normal. When cirrhosis develops, sonography may demonstrate a coarsened echotexture and other morphologic changes of cirrhosis. The World Health Organization (WHO) defines cirrhosis as a diffuse process characterized by fibrosis and the conversion of normal liver architecture into structurally abnormal nodules. (Anthony PP et.al 1977).

Three major pathologic mechanisms combine to create cirrhosis: cell death, fibrosis, and regeneration. Cirrhosis has been classified as micronodular, in which nodules are 0.1 to $1 \mathrm{~cm}$ in diameter, and macronodular, characterized by nodules of varying size, up to $5 \mathrm{~cm}$ in diameter. Consumption is the most common cause of micro-nodular cirrhosis, and chronic viral hepatitis is the most frequent cause of the macronodular form. (Sadler GH. et.al 1987) Patients who continue to drink may go on to end-stage liver disease, which is indistinguishable from cirrhosis of other causes. Other etiologies are biliary cirrhosis (primary and secondary), Wilson's disease, primary sclerosing cholangitis, and hemochromatosis. The classic clinical presentation of cirrhosis is hepatomegaly, jaundice, and ascites. However, serious liver injury may be present without any clinical clues. In fact, only $60 \%$ of patients with cirrhosis have signs and symptoms of liver disease. The sonographic appearance associated with cirrhosis include the following;

Volume redistribution. Several studies have evaluated the ratio of the caudate lobe width to the right lobe width $(\mathrm{C} / \mathrm{RL})$ as an indicator of cirrhosis. (Giorgio A, et.al 1986)

A C/RL value of 0.65 is considered indicative of cirrhosis. The specificity is high $(100 \%)$, but the sensitivity is low (43\%-84\%), indicating that the $\mathrm{C} / \mathrm{RL}$ ratio is a useful measurement if it is abnormal. (Giorgio A, et.al 1986)

Coarse echotexture. Increased echogenicity and coarse echotexture are frequent observations in diffuse liver disease.

Nodular surface. Irregularity of the liver surface during routine scanning has been appreciated as a sign of cirrhosis when the appearance is gross or when ascites is present. (Freeman MP et.al 1986). Regenerating nodules (RNs). These regenerating hepatocytes are surrounded by fibrotic septae. Because RNs have a similar architecture to the normal liver, ultrasound and CT have limited ability in their detection. RNs tend to be isoechoic or hypoechoic with a thin, echogenic border that corresponds to fibrofatty connective tissue. (Freeman MP et.al 1986). MRI has a greater sensitivity than both $\mathrm{CT}$ and ultrasound in $\mathrm{RN}$ detection. Because some RNs contain iron, gradient echo sequences demonstrate these nodules as hypointense. Murakami T et.al (1990). Dysplastic nodules. Dysplastic nodules or adenomatous hyperplastic nodules are larger than RNs (diameter of $10 \mathrm{~mm}$ ) and are considered premalignant. (Theise ND (1995). they contain well-differentiated hepatocytes, a portal venous blood supply, and atypical or frankly malignant cells. This study was aimed to assess and to characterize liver disease (hepatitis $(\mathrm{B}, \mathrm{C})$, liver cirrhosis and normal liver) by using of image texture analysis by introducing a new method of computer aided diagnosis of liver disease. Generally Chronic liver disease (CLD) has various etiologies, with the viral infection of hepatitis $\mathrm{B}$ virus (HBV), hepatitis $\mathrm{C}$ virus (HCV), human immunodeficiency virus, alcohol consumption, hepatotoxic drug ingestion, nonalcoholic fatty liver, autoimmune diseases, and cryptogenic hepatopathy being commonly encountered in daily practice. Histologically, liver fibrosis develops and gradually progresses as a result of following a wound-healing response in patients with CLD. In particular, activation of cellular elements including myofibroblasts and stellate cells results in collagen deposition and subsequent development of CLD (Friedman SL.2008) (Talwalkar JA. 2008). Liver biopsy is known as the gold standard for diagnosing liver fibrosis.

Hassan A. B, et.al (2016) stated that the texture analysis is an essential issue in image processing. It comprises a set of mathematical techniques used to quantify the different gray levels within an image in terms of intensity and distribution. Texture represents the spatial arrangement of pixels' gray levels in a region. In this study we used FOS, First-order texture analysis measures use the image histogram, or pixel occurrence probability, to calculate texture. In general seven features commonly used to describe the properties of the image histogram, and therefore image texture, are computed. These are: mean; variance; coarseness; skewness; kurtosis; energy; and entropy as stated by Nailon, W.H. (1997).

\section{Material and Method}

This study was aimed to classify the liver disease (cirrhosis, hepatitis and normal liver in ultrasound images using image texture analysis by using of image processing program called (interactive data language, IDL) firstly patient with differential diagnosis of these selected liver disease was underwent successful laboratory and imaging investigation particularly for those suffering from RUQ pain and associated symptoms, then the liver was examined with realtime sonography, by using of mobile GE LOGIQ 5 ultrasound machine, ideally after a 6-hour fast. Both supine and right anterior oblique views should be obtained. Sagittal, transverse, coronal, and subcostal oblique views are suggested using both a standard abdominal transducer and a higher frequency transducer. Many patients' liver is tucked beneath the lower right ribs, so a transducer with a small scanning face, allowing an intercostal approach, is invaluable. (Wilson SR, et.al 2009) Furthermore we sometimes using of volumetric imaging to ultrasound contributes greatly to the evaluation of the liver as a single, appropriately selected acquisition and may show virtually the entire liver, allowing for a rapid portrayal of liver anatomy, size, texture, and surface characteristics. Therefore, differentiation of the diffuse changes of cirrhosis and fatty liver from normal are enhanced by review of the videos.

Using these diagnostic criteria the US images was extracted and treated as IDL variables (tiff format), preparing for feature extraction; the image was displayed on IDL disc as gray scale image then proper enhancement was undertaken using smoothing function, and by using of $(3 \times 3$ pixel $)$

\section{Volume 5 Issue 2, February 2016}




\section{International Journal of Science and Research (IJSR) \\ ISSN (Online): 2319-7064 \\ Index Copernicus Value (2013): 6.14 | Impact Factor (2014): 5.611}

window the image was scanned and the feature of the FOS was calculated as:

Let random variable $I$ represents the gray levels of image region. The first-order histogram $P(I)$ is defined as:

$$
P(I)=\frac{\text { number of pixels with gray level } I}{\text { total number of pixels in the region }}
$$

Based on the definition of $P(I)$, the Mean $m 1$ and Central Moments $\mu k$ of $I$ are given by:

$$
\begin{gathered}
m_{1}=E\left[I^{1}\right]=\sum_{I=0}^{N_{g}-1} I^{1} P(I) \\
\mu_{k}=E\left[(I-E[I])^{k}\right]=\sum_{I=0}^{N_{g}-1}\left(I-m_{1}\right)^{k} P(I), \\
k=2,3,4
\end{gathered}
$$

Where $\mathrm{Ng}$ is the number of possible gray levels.

The most frequently used central moments are Variance, Skewness and Kurtosis given by $\mu 2, \mu 3$, and $\mu 4$ respectively. The Variance is a measure of the histogram width that measures the deviation of gray levels from the Mean. Skewness is a measure of the degree of histogram asymmetry around the Mean and Kurtosis is a measure of the histogram sharpness. As stated by Aggarwal and Agrawal (2012).

These parameters was extracted for liver cirrhosis, hepatitis $\mathrm{B}$, and $\mathrm{C}$ relative to the normal echo texture of live ultrasound as control group. Then using linear discriminant function with step-wise technique the classification and discrimination was performed and the result as follow:

\section{Result Presentation}

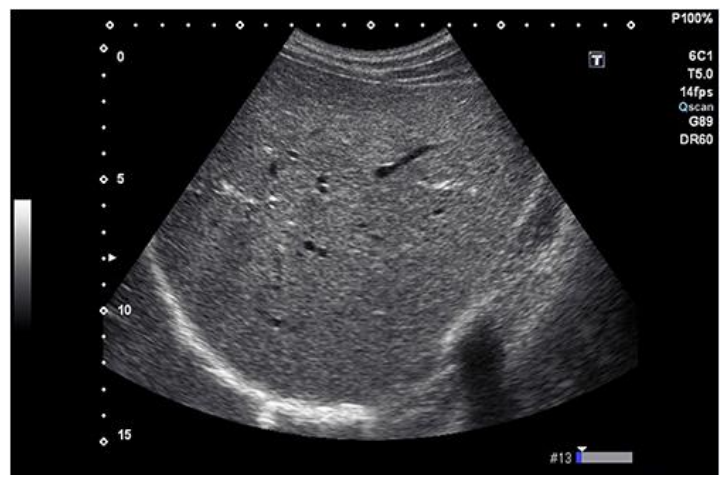

Figure 2: Normal liver ultrasonographic image

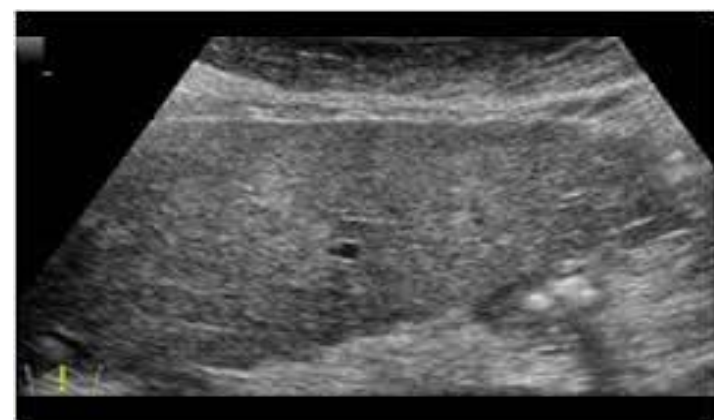

Figure 3: liver cirrhosis ultrasound
Table 1: Showed the classification function using linear discrimination analysis

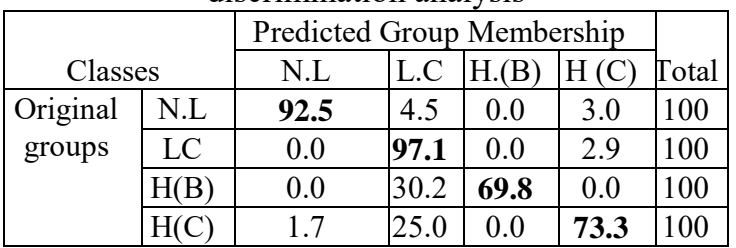

$84.9 \%$ of original grouped cases correctly classified. N.L: Normal Liver

L.C: Liver Cirrhosis

H. (B): Hepatitis (B)

H. (C): Hepatitis (C)

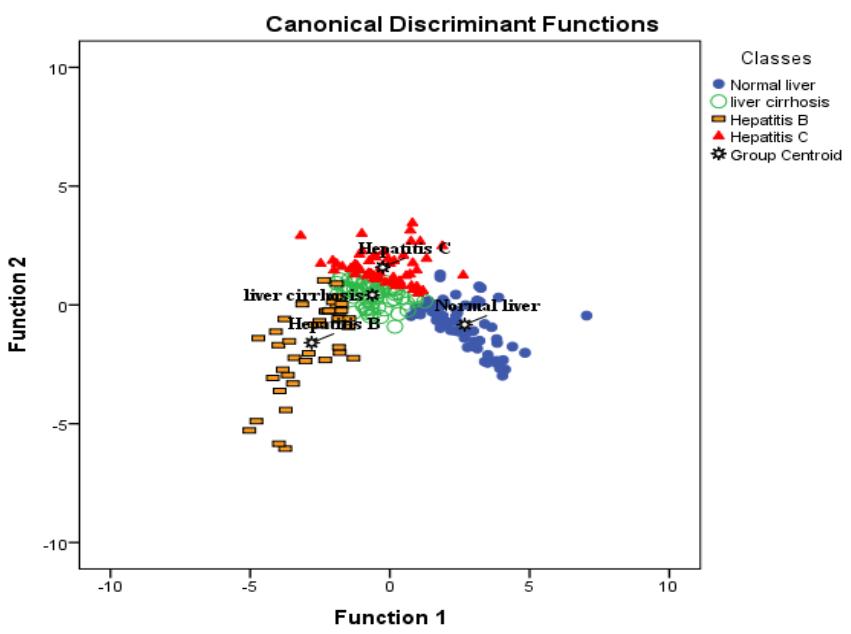

Figure 4: Scatter plot generated using discriminate analysis function for four classes represents: NL, LC, H (B) and $\mathrm{H}$ (C).

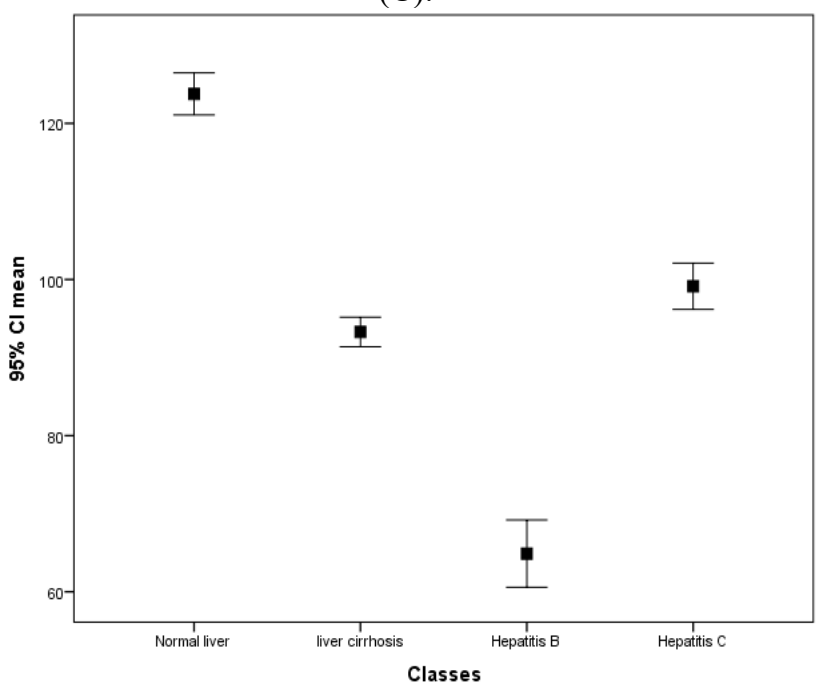

Figure 5: Classification based on mean for US images. 


\section{International Journal of Science and Research (IJSR) \\ ISSN (Online): 2319-7064}

Index Copernicus Value (2013): 6.14 | Impact Factor (2014): 5.611

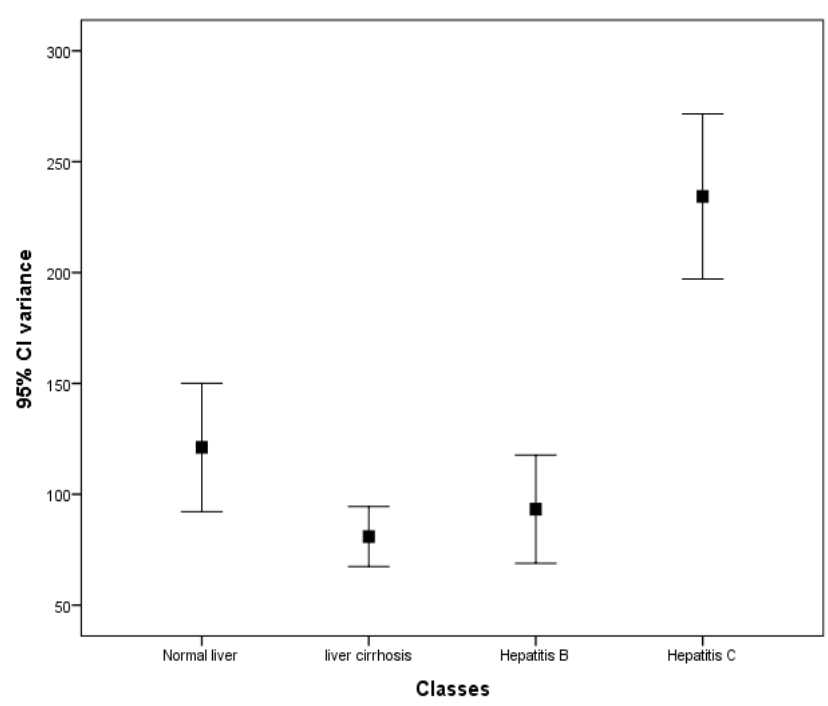

Figure 6: Classification based on variance for US images.

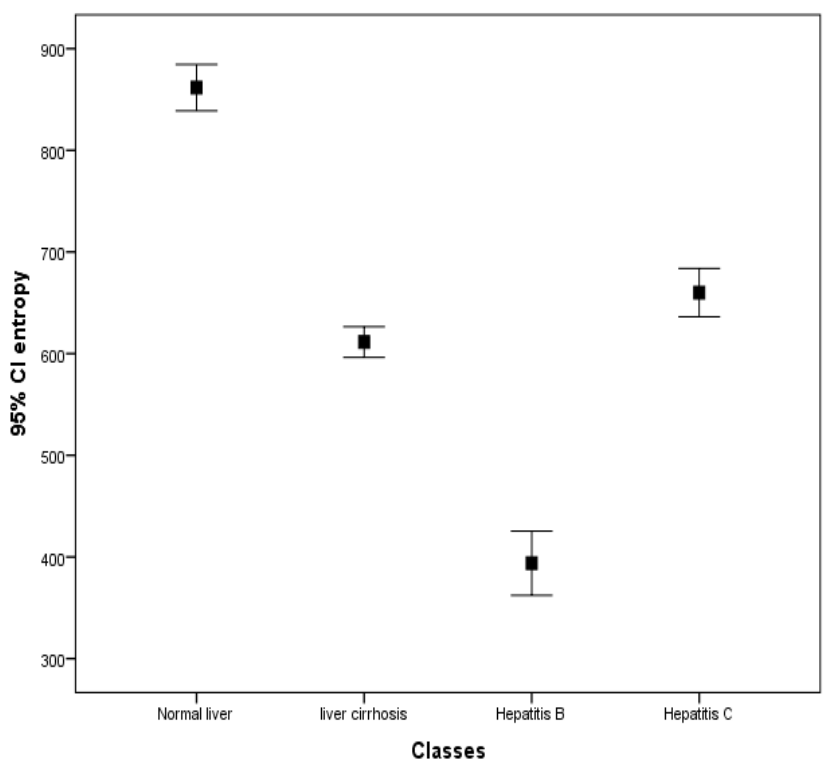

Figure 7: Classification based on entropy for US images.

\section{Discussion}

Much of our understanding of machine vision algorithms is a result of attempts to overcome the failings of the human visual system to detect certain textured patterns. This understanding has proven vital in evaluating and comparing the performance of human vision against machine-based texture analysis approaches. Julesz, an experimental psychologist, was an early pioneer in the visual perception of texture (Julesz, 1975). And here we introducing a new method for texture classification rather than using of normal human vision properties. Firstly the classification aimed to extract these feature from the image based on the normal image histogram where the primary image (figure 1.) was converted into tiff format as an input image for IDL image processing program, then a window of $3 \times 3$ was created in order to scan the image then the feature were extracted for three different phases of CT series; mean; variance; coarseness; skewness; kurtosis; energy; and entropy. All these feature were calculated for all images with many different pathology particularly for patient who have liver cirrhosis, hepatitis B and C comparing to the normal liver texture and then the data were ready for discrimination which was performed using step-wise technique in order to select the most significant feature that can be used to classify these liver disease and the result showed that: Table (1). Linear discriminant function and the classification accuracy of each class was presented in which $84.9 \%$ of liver tissue and disease was correctly classified and $92.5 \%, 97.1 \%, 69.8 \%$ and $73.3 \%$ classification sensitivity for normal liver, liver cirrhosis, hepatitis $\mathrm{B}$ and $\mathrm{C}$ respectively with classification specificity of normal liver tissue equal to $80.06 \%$ were computed from gray level histogram and the results are represented that there is a well concentration of features around the class centers which give a remarkable difference among the four classes. (Fig 4.).

Figure 5: demonstrate the classification of these disease based on the mean textural feature which used to measure the pixel distribution on its homogeneity on the selected classes this figure reveals that the normal liver is discriminated better from the hepatitis B while the mean difference between the hepatitis $\mathrm{C}$ and liver cirrhosis was not big any more. Low mean value and range for hepatitis $B$ and very high value for normal liver texture.

Figure 6: showed the classification that was based on the variance which is considered as one of the significant feature that selected to classify these lesions which used to measure the deviation of pixel intercity from the mean value, this texture classify the hepatitis $\mathrm{C}$ more better than other selected feature from other liver pathology.

Entropy have same result to the mean feature, as demonstrated in figure 7.

Finally, excellent discrimination between normal liver texture and hepatitis B, C can be established on the basis as few as three optimal feature among the many different texture characteristics tested [FOS]. This serves as a second method to perform more characterization of the hepatitis, normal liver and other liver pathological disturbance because many liver disease causing progressive changes in liver cellular properties.

From survey on the previous published studies we noted that using of small pixel window to scan the MR, CT, and US images is better than using bigger windows because of byte scale images and its small dynamic range of the pixel variation. (Hassan A. B et.al 2016).

\section{Conclusion}

This study conclude that normal liver and pathological liver disease are classified better on US images for simplicity can be diagnosed and classify by using the following simple equation after extracting the associated features using a window of $3 \times 3$ pixel from the region of interest; the biggest classification score assume the tissue type:

The following equations that can be used for future texture estimation and liver disease charactrization without need for 


\section{International Journal of Science and Research (IJSR) \\ ISSN (Online): 2319-7064}

Index Copernicus Value (2013): 6.14 | Impact Factor (2014): 5.611

further textural calculation:

\begin{tabular}{l} 
Normal liver $=($ mean $\times 48.6)+($ variance $\times 0.16)+($ energy $\times$ \\
$0.29)+($ entropy $\times-5.10)-453.52$ \\
Liver cirrhosis $=($ mean $\times 51.31)+($ variance $\times 0.17)+($ energy $\times$ \\
$0.36)+($ entropy $\times-6.35)-418.29$ \\
\hline Hepatitis $B=($ mean $\times 48.1)+($ variance $\times 0.15)+($ energy $\times 0.34)$ \\
$+($ entropy $\times-5.98)-412.72$ \\
Hepatitis $C=($ mean $\times 52.03)+($ variance $\times 0.18)+($ energy $\times$ \\
$0.38)+($ entropy $\times-6.43)-502.72$
\end{tabular}

\section{References}

[1] Abdoelrahman Hassan A. B., Samia A. F. Ahmed, M.E.M Gar-elnabi, M. A. Ali Omer, Asma I. Ahmed, Characterization of Hepatocellular Carcinoma (HCC) in CT Images using Texture Analysis Technique, IJSR, 2016; v.5; 917: 921

[2] Theise ND. Macroregenerative (dysplastic) nodules and hepato-carcinogenesis: theoretical and clinical considerations. Semin Liver Dis 1995; 15: 360-371.

[3] Murakami T, Kuroda C, Marukawa T, et al. Regenerating nodules in hepatic cirrhosis: MR findings with pathologic correlation. AJR Am J Roentgenol 1990; 155: 1227-1231.)

[4] Freeman MP, Vick CW, Taylor KJ, Carithers RL, Brewer WH. Regenerating nodules in cirrhosis: sonographic appearance with anatomic correlation. AJR Am J Roentgenol 1986; 146: 533-536.)

[5] Giorgio A, Amoroso P, Lettieri G, et al. Cirrhosis: value of caudate to right lobe ratio in diagnosis with ultrasound. Radiology 1986; 161:443-445.)

[6] Millward-Sadler GH. Cirrhosis. In: MacSween RNM, Anthony PP, Scheuer PJ, editors. Pathology of the liver. 2nd Ed. New York: Churchill Livingstone; 1987. p. 342363.)

[7] Talwalkar JA. Elastography for detecting hepatic fibrosis: options and considerations. Gastroenterology 2008; 135: 299-302.

[8] Friedman SL. Mechanisms of hepatic fibro genesis. Gastroenterology 2008; 134: 1655-1669.

[9] Anthony PP, Ishak KG, Nayak $\mathrm{NC}$, et al. The morphology of cirrhosis: definition, nomenclature, and classification. Bull WHO 1977; 55: 521-540.)

[10]Zwiebel WJ. Sonographic diagnosis of diffuse liver disease. Semin Ultrasound CT MR 1995; 16: 8-15).

[11] Wilson SR. The gallbladder. In: Gastrointestinal disease. 6th series. Test and syllabus. Reston, Va: American College of Radiology; 2004.).

[12] Davis GL. Chronic hepatitis. In: Kaplowitz N, editor. Liver and biliary disease. 2nd ed. Baltimore: Williams \& Wilkins; 1996. p. 327-337.)

[13] Niederau C, Sonnenberg A, Muller JE, et al. Sonographic measurements of the normal liver, spleen, pancreas, and portal vein. Radiology 1983; 149:537540)

[14] Gosink BB, Leymaster CE. Ultrasonic determination of hepatomegaly. J Clin Ultrasound 1981; 9: 37-44.

[15] Sugarbaker PH: Toward a standard of nomenclature for surgical anatomy of the liver. Neth J Surg 1988; PO: 100.)
[16] Soyer P, Bluemke DA, Bliss DF, et al. Surgical segmental anatomy of the liver: demonstration with spiral CT during arterial portography and multiplanar reconstruction. AJR Am J Roentgenol 1994; 163:99103.)

[17]Nailon, W.H. (1997). Tissue characterisation of intravascular ultrasound using texture analysis. Ph.D. dissertation, The University of Edinburgh, School of Engineering.

[18] Namita Aggarwal, R. K. Agrawal, First and Second Order Statistics Features for Classification of Magnetic Resonance Brain Images, Journal of Signal and Information Processing, 2012, 3, 146-153.

[19] Wilson SR, Gupta C, Eliasziw M, Andrew A. Volume imaging in the abdomen with ultrasound: how we do it. AJR Am J Roentgenol 2009;193: 79-85.

[20] Julesz, B. (1975). Experiments in the visual perception of texture. Scientific American, Vol. 232, pp 34-43.

\section{Author Profile}

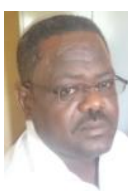

Mr. Babiker Abd Elwahab Awad Allah (Sudan) received the B.Sc. degree in diagnostic radiology technology, COMRS, Sudan university of science and technology, 2000, as well as higher diploma and M.Sc. in medical diagnostic ultrasound in 2003, and 2009 respectively, he was working as lecturer at college of medical radiologic science since 2003, as well as sonlogist in modern medical center, ultrasound department at college of medical radiologic, now he is a $\mathrm{PhD}$ student in medical diagnostic ultrasound, and he has been active in ultrasound and diagnostic radiology researches.

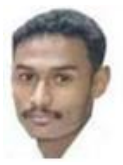

Mr. Abdoelrahman Hassan Ali Bakry (Sudan) received the (B.Sc.) and (M.Sc.) in radiotherapy technology from College of Medical radiological Science, Sudan University of Science and Technology in 2013 and 2015 respectively. During 2013 up to date, he is staying in College of Medical radiological Science, Sudan University of Science and Technology, Radiology Department, Antalya Medical Center and Elnileen Diagnostic Medical Center; also he has been active in Computerized Texture Analysis, Radiotherapy-Oncology, and diagnostic radiology, Medical physics, ultrasound and Nuclear Medicine researches. Now he is assistant teacher at SUST also (2014).

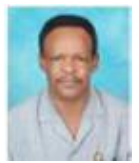

Assoc. proff. Dr. Mohamed Elfadil Mohamed Garelnabi (Sudan) awarded the B. Sc. in Radiotherapy and Nuclear Medicine (1987) and M.Sc. in Radiation Therapy (2000-SUST) and Ph. D. degree in Medical Physics (Natal University-South Africa) in 2007. During 1996-2012 he has been working as lecturer as well as Associate Prof. at SUST department of Radiation therapy. Also he has been active in Computerized Texture Analysis, RadiotherapyOncology, Ultrasound and Nuclear Medicine researches.

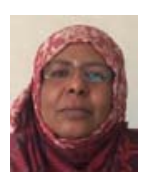

Assistant. Professor. Dr. Asma Ibrahim Ahmed (Sudan), awarded the B.Sc. in diagnostic radiology technology (1996), M.Sc. in medical ultrasound (2005, SUST), as well as $\mathrm{PhD}$ degree in diagnostic radiology (SUST, 2012), during 1998 she has been working as teaching assistant at COMRS, SUST, as well as lecturer, and assistant professor in 2005, and 2012 respectively. She has been active in diagnostic radiology, medical ultrasound researches. 